\title{
ALMOST PERIODIC OPERATORS OF INNER AUTOMORPHISM GROUPS
}

\author{
F. JAVIER THAYER
}

\begin{abstract}
We examine the almost periodic part of the inner automorphism group of $\mathfrak{B}(H)$ arising from a representation of a locally compact abelian group.
\end{abstract}

1. Introduction and notation. Let $G$ be a locally compact group, and $\pi_{1}, \pi_{2}$ representations of $G$ on Hilbert spaces $W_{1}, W_{2}$, respectively. For $g \in G$ and $t$ $\in \mathscr{B}\left(W_{1}, W_{2}\right)$, let $\alpha(g) t=\pi_{2}(g) t \pi_{1}(g)^{*}$. In [1] Arveson considers, for certain representations of locally compact abelian groups on Banach spaces (including the representation $\alpha$ just described), a general notion of spectral subspace corresponding to a closed subset of the dual group. This generalizes the concept of spectral projection for representations on Hilbert spaces. In this note we extend these ideas somewhat considering the notions of containment and disjointness for Banach space representations. Our interest in this is the problem of determining the finite-dimensional representations contained in $\alpha$. This essentially is asking for the structure of the almost periodic part of $\alpha$, i.e. the subspace of $\{t: \alpha(G) t$ is norm relatively compact $\}$. For example if $\pi_{1}=\pi_{2}=\pi$ is the representation induced by a measure preserving action of $G$ on a measure space $(X, \mu)$ and $\pi$ has pure point spectrum, then the set of almost periodic multiplication operators is weakly dense in the algebra of all multiplications by bounded measurable functions. However is it possible to have no almost periodic operators at all other than those in $\pi(G)^{\prime}$ ? We give an example to show this may occur if the spectral measure of $\pi$ is sufficiently nonsymmetric.

We refer to $[2,5.2,5.3,8.4]$ for the definitions and basic properties of disjointness, quasi-equivalence and spectral measures of representations of groups on Hilbert spaces.

If $G$ is a topological group (we will henceforth refer only to "group"), a representation of $G$ on a Banach space $V$ will be a morphism $\rho$ of $G$ into the group of invertible contractions (ipso facto isometries) of $\mathscr{B}(V, V)$ with the following continuity requirement (related to hypothesis 1.5 in [1]): there is a coarser locally convex topology $\tau$ on $V$ such that $\rho$ is continuous if $\mathscr{B}(V, V)$ has the simple $\tau$-convergence topology [in nets: $t_{\alpha} \rightarrow t$ iff $t_{\alpha} \xi \rightarrow t \xi$ in $\tau$ for all $\xi \in V]$. We will use this condition in essentially only one place [Lemma 1]. If

Received by the editors April 14, 1976.

AMS (MOS) subject classifications (1970). Primary 46L05; Secondary 43A60, 47D10. 
$V$ is a Hilbert space this continuity condition is equivalent to strong continuity. If no confusion should arise we will say $G$ acts on $V$ omitting the datum $\pi$.

If $G$ acts on $W_{i}, i=1, \ldots, n+1$, then the space of $n$-linear maps $W_{1} \times \cdots \times W_{n} \rightarrow W_{n+1}$, which we denote by $\mathscr{B}\left(W_{1} ; \ldots ; W_{n}, W_{n+1}\right)$, carries the canonical $G$ action

$$
(g \cdot \phi)\left(\xi_{1}, \ldots, \xi_{n}\right)=g \phi\left(g^{-1} \xi_{1}, \ldots, g^{-1} \xi_{n}\right)
$$

If $\tau$ is a locally convex topology on $W_{n+1}$ fulfilling the continuity requirement, then it is a straightforward verification that simple $\tau$ convergence in $\mathfrak{B}\left(W_{1} ; \ldots ; W_{n}, W_{n+1}\right)$ fulfills the continuity requirement for the induced action.

We denote the space of elements in $\Re_{(}\left(W_{1} ; \ldots ; W_{n}, W_{n+1}\right)$ fixed under this action by $\mathscr{B}_{G}\left(W_{1} ; \ldots ; W_{n}, W_{n+1}\right)$; we refer to its elements as the $G$ intertwining $n$-linear maps. Observe there is a canonical isomorphism $\mathscr{B}_{G}\left(W_{1} ; W_{2}, W_{3}\right)$ $\rightarrow \mathscr{B}_{G}\left(W_{1}, \mathfrak{B}\left(W_{2}, W_{3}\right)\right)$ given by $b \mapsto\left\{\xi_{1} \mapsto\left\{\xi_{2} \mapsto b\left(\xi_{1}, \xi_{2}\right)\right\}\right\}$.

If $\pi_{i}, i=1,2$, are representations of $G$ on $W_{i}, i=1,2$, and $W_{1} \widetilde{\otimes} W_{2}$ is the complete projective tensor product [6, III, 6.3], then there is a unique representation $\pi_{1} \otimes \pi_{2}$ of $G$ on $W_{1} \widetilde{\otimes} W_{2}$ with the conditions $\left(\pi_{1} \otimes \pi_{2}(g)\right)$ $\cdot\left(\xi_{1} \otimes \xi_{2}\right)=\pi_{1}(g) \xi_{1} \otimes \pi_{2}(g) \xi_{2}$. It is straightforward there is a unique representation of the discrete version $G^{d}$ of $G$ with this property. If $\tau_{1}, \tau_{2}$ are locally convex topologies on $W_{1}, W_{2}$, respectively, which fulfill the continuity requirement defined by seminorms $\left\{p_{\alpha}^{1}\right\},\left\{p_{\beta}^{2}\right\}$, then the seminorms $p_{\alpha}^{1} \otimes p_{\beta}^{2}[6$, III, 6.3] extend uniquely to continuous seminorms on $W_{1} \widetilde{\otimes} W_{2}$. It is easily verified that this topology indeed satisfies the continuity requirement.

\section{Spectral subspaces.}

Definition 1. Let $\pi_{i}, i=1,2$, be representations of the group $G$ on $W_{i}, i=1,2$, respectively. We say $\pi_{1}$ is disjoint going into $\pi_{2}\left(\pi_{1} \phi \pi_{2}\right)$ iff

$$
\mathscr{B}_{G}\left(W_{1}, W_{2}\right)=(0) \text {. }
$$

We denote by $S\left(\pi_{1} ; \pi_{2}\right)$ (or $S\left(\pi_{1}\right)$ if $\pi_{2}$ is understood) the closed linear span of the sets $t\left(W_{1}\right)$ for $t \in \mathscr{B}_{G}\left(W_{1}, W_{2}\right)$. This is somewhat different from Arveson's definition of spectral subspace.

Proposition 1. Let $V, W, Y$ be Banach spaces $\pi_{W}, \pi_{V}, \pi_{Y}$ representations of $G$ on $V, W, Y$, respectively. Then the following are equivalent:

(a) $V \phi \mathfrak{B}(W, Y)$.

(b) There is no nonzero $b \in \mathscr{B}_{G}(V ; W, Y)$.

(c) $\pi_{V} \otimes \pi_{W} \phi \pi_{Y}$.

Proof. (a) is equivalent to $\mathscr{B}_{G}(V, \mathfrak{B}(W, Y)) \cong \mathscr{B}_{G}(V ; W, Y)=\{0\}$ which is (b). On the other hand (c) is equivalent to $\mathscr{B}_{G}(V \bar{\otimes} W, Y) \cong \mathscr{B}_{G}(V ; W, Y)$ $=\{0\}$ which is $(\mathrm{b})$.

Definition 2. If the conditions of Proposition 1 hold then we say $\left(\pi_{V}, \pi_{W}, \pi_{Y}\right)$ is a singular triple. 
In the following we consider $G$ acting on $\mathscr{B}(W, Y) ; S\left(\pi_{V}\right)$ refers to this action.

Proposition 2. Suppose $\pi_{1}, \pi_{2}$ are representations of $G$ on the Banach space $W, E$ a projection such that $\operatorname{Ran} E$ is $\pi_{1}$ invariant, $\operatorname{Ran}(1-E)$ is $\pi_{2}$ invariant. If $\pi_{V}$ is a representation of $G$ on the Banach space $V, \operatorname{Ran} E$ is $S\left(\pi_{V}\right)$ invariant iff $\left(\pi_{V},\left.\pi_{1}\right|_{\operatorname{Ran} E},\left.\pi_{2}\right|_{\operatorname{Ran}(1-E)}\right)$ is singular.

Proof. Denote by $W_{1}, W_{2}$ the Banach space $W$ with the action $\pi_{1}, \pi_{2}$, respectively. Now $\operatorname{Ran} E$ is $S\left(\pi_{V}\right)$ invariant iff for all $t \in \mathscr{B}_{G}\left(V, \mathscr{B}\left(W^{1}, W^{2}\right)\right)$, $(1-E) t(V) E=0$. This means there is no $t \neq 0$ such that

$$
t \in \mathscr{B}_{G}(V, \mathscr{B}(\operatorname{Ran} E, \operatorname{Ran}(1-E)))
$$

where $G$ acts on the subspaces $\operatorname{Ran} E, \operatorname{Ran}(1-E)$ by $\left.\pi_{1}\right|_{\operatorname{Ran} E},\left.\pi_{2}\right|_{\operatorname{Ran}(1-E)}$ respectively. This is the condition $\left(\pi_{V},\left.\pi_{1}\right|_{\operatorname{Ran} E},\left.\pi_{2}\right|_{\operatorname{Ran}(1-E)}\right)$ be singular.

If $G$ is a separable type 1 locally compact group, $\pi$ a unitary representation of $G$ on a separable Hilbert space $H$, there is a Borel measure $\mu_{\pi}$ on the dual $G^{\wedge}$ of $G[2,7.2 .1]$ unique up to equivalence such that $\pi \approx \int_{G^{\wedge}}^{\oplus} \phi d \mu_{\pi}(\phi)$ where $\approx$ denotes quasi-equivalence. $\pi_{1} \phi \pi_{2}$ iff $\mu_{\pi_{1}}, \mu_{\pi_{2}}$ are mutually singular. If we denote by $\pi_{1} \otimes_{i} \pi_{2}$ the inner tensor product of $\pi_{1}, \pi_{2}$ (acting on the Hilbert space tensor product of the corresponding spaces), then for $G$ commutative it is known that $\mu_{\pi_{1} \otimes \pi_{2}} \equiv \mu_{\pi_{1}} * \mu_{\pi_{2}}$ where ' $\equiv$ ' is equivalence \{see [4, Proposition 2.11] for the case $G=R^{n}$ although essentially the same reasoning is correct in the general case $)$. Also if $G$ is commutative $\pi(G)^{\prime \prime} \cong L^{\infty}\left(G^{\wedge}, \mu_{\pi}\right)$ so that we may associate to each selfadjoint projection $E \in \pi(G)^{\prime \prime}$ a unique (up to null sets) Borel set $\tau(\pi, E)$ such that the image of $E$ is the indicator function of $\tau(\pi, E)$. If $E$ is a s.a. projection in $\pi(G)^{\prime \prime}$ then $\left.\mu_{(\pi \mid E)} \equiv \mu_{\pi}\right|_{\tau(\pi, E)}$. Finally if $E$ is a s.a. projection in $\pi(G)^{\prime}$ and $F$ is its central cover then $\left.\left.\pi\right|_{F} \approx \pi\right|_{E}$. With these remarks we will prove the following proposition.

Proposition 3. Let $G$ be a separable L.C.A. group, $\pi_{1}, \pi_{2}$ representations of $G$ on the space $W$. Then if $E \in \pi_{1}(G)^{\prime} \cap \pi_{2}(G)^{\prime}$ is a s.a. projection and $\pi_{V}$ is a representation of $G$ on the finite-dimensional Hilbert space $V, \operatorname{Ran} E$ is $S\left(\pi_{V}\right)$ invariant iff

$$
\left.\mu_{\pi_{V}} * \mu_{\pi_{1}}\right|_{\tau\left(\pi_{1}, F\right)},\left.\quad \mu_{\pi_{2}}\right|_{\tau\left(\pi_{2}, H\right)}
$$

are singular where $F, H$ are the central covers of $E, 1-E$ in $\pi_{1}(G)^{\prime}, \pi_{2}(G)^{\prime}$, respectively.

Proof. By the remarks preceding the proposition we have the equivalences:

$$
\begin{aligned}
\mu_{\pi_{V} \otimes_{i}\left(\pi_{1} \mid E\right)} & \left.\left.\equiv \mu_{\pi_{V}} * \mu_{\pi_{1}}\right|_{\tau\left(\pi_{1} \mid F\right)} \equiv \mu_{\pi_{V}} * \mu_{\pi_{1}}\right|_{\tau\left(\pi_{1}, F\right)}, \\
\mu_{\left(\pi_{2} \mid 1-E\right)} & \left.\equiv \mu_{\pi_{2}}\right|_{\tau\left(\pi_{2}, H\right)}
\end{aligned}
$$

Thus $\pi_{V} \otimes_{i}\left(\pi_{1} \mid E\right), \pi_{2} \mid 1-E$ are disjoint iff the measures from the two equiva- 
lences are disjoint. By Proposition 2 and the fact that the Hilbert space norm and the projective norm on the algebraic tensor poduct $V \otimes W$ are complete and coincide because $V$ is finite dimensional, we conclude the proof.

3. Almost periodicity. If $G$ is a topological group, $\pi_{1}, \pi_{2}$ representations of $G$ on a Banach space $W$, then we have seen $\alpha(g) t=\pi_{2}(t) t \pi_{1}(g)^{-1}$ is a representation of $G$ on $\mathscr{B}(W)$; the strong operator topology on $\mathscr{B}(W)$ fulfills the continuity requirement. We now investigate the set of operators for which the orbit $\alpha(G) t$ is relatively norm compact, i.e. is $\alpha$-almost periodic. For clarity of presentation we give a definition and lemma in the general context of $G$ actions on Banach spaces. The lemma is surely known although no direct reference is available to us.

Definition 3. Let $G$ be a topological group, $\pi$ a representation of $G$ on the Banach space $W$. An element $x \in W$ is almost periodic iff $\pi(G) x$ is norm relatively compact.

Lemma 1. Let $G, \pi, W$ be as above. If $W^{0}$ denotes the set of almost periodic vectors then, $W^{0}$ is a norm closed subset and is the closed linear span of the spaces $S(\lambda), \lambda$ a finite-dimensional unitary representation of $G$.

Proof. That $W^{0}$ is a norm closed subspace follows readily from the definitions. If $\sigma: G^{d} \rightarrow \Sigma\left(G^{d}\right)$ is the Bohr compactification of the discrete version $G^{d}$ of $G$, then there is a unique morphism $\sigma(\pi): \Sigma\left(G^{d}\right) \rightarrow \mathscr{B}\left(W^{0}\right)$ so that the diagram

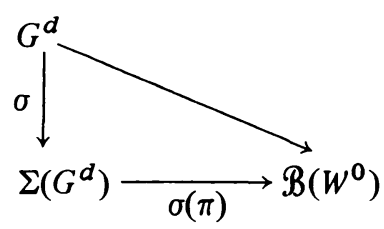

commutes and which is continuous if $\mathscr{B}\left(W^{0}\right)$ has the simple norm convergence topology. This is just the universal property for the Bohr compactification [2, 16.1.1]. Now it is known that if $\rho$ is a strongly continuous representation of a compact group on a Banach space $V$, then $V$ is the closed linear span of the finite-dimensional $\rho$ irreducible subsets of $V$. (For $V$ a Hilbert space this is a basic result in functional analysis. For Banach spaces and even certain locally convex spaces the result remains correct. Consult the notes [3].) Thus $W^{0}$ is the closed linear span of the finite-dimensional $\pi$ irreducible subspaces of $W$. Observe that if $U \subseteq W$ is finite dimensional and $\pi$ invariant, then $\left.\pi\right|_{U}$ is continuous in the simple norm convergence topology. (This follows from the continuity requirement and the fact that all Hausdorff vector space topologies coincide on $U$.) Now any finite-dimensional representation of $G$ is equivalent to a unitary one, so the lemma follows.

Proposition 4. Suppose $\pi_{1}, \pi_{2}$ are representations of $G$ on the Banach space $W, \mathscr{B}(W)^{0}$ the set of operators almost periodic under the action $t$ 
$\mapsto \pi_{2}(g) t \pi_{1}(g)^{-1}$. If $E$ is a projection such that $\operatorname{Ran} E$ is $\pi_{1}$ invariant, $\operatorname{Ran}(1-E)$ is $\pi_{2}$ invariant, then $\operatorname{Ran} E$ is $\mathscr{B}(W)^{0}$ invariant iff $\left(\lambda,\left.\pi_{1}\right|_{\operatorname{Ran} E}\right.$, $\left.\left.\pi_{2}\right|_{\operatorname{Ran} 1-E}\right)$ is singular for all finite-dimensional irreducible unitary representations $\lambda$ of $G$.

Proof. The statement is an immediate consequence of Proposition 2 and the previous lemma.

Proposition 5. Let $G$ be a separable L.C.A. group, $\pi_{1}, \pi_{2}$ representations of $G$ on the separable Hilbert space $W$. Then if $E \in \pi_{1}(G)^{\prime} \cap \pi_{2}(G)^{\prime}$ is a s.a. projection, $E$ is $\mathscr{B}(W)^{0}$ invariant iff

$$
{ }_{\lambda}\left(\left.\mu_{\pi_{1}}\right|_{\tau\left(\pi_{1}, F\right)}\right),\left.\quad \mu_{\pi_{2}}\right|_{\tau\left(\pi_{2}, H\right)}
$$

are singular for all $\lambda \in G^{\hat{\prime}}$, where $F, H$ are the central covers of $E, 1$ - $E$ in $\pi_{1}(G)^{\prime}, \pi_{2}(G)^{\prime}$, respectively.

Proof. This is a direct consequence of Proposition 3 and the lemma. Notice the convention for the translate of $\nu$ is $\lambda^{\nu}=\varepsilon_{\lambda} * \nu$.

4. The commutant of $\mathscr{B}(W)^{0}$. At this point we restrict attention to inner automorphism groups $\alpha$ : $g \mapsto\left\{t \mapsto \pi(g) t \pi(g)^{*}\right\}$ where $G$ is separable L.C.A. and $\pi$ a representation of $G$ on a separable Hilbert space $W$.

Proposition 6. Let $\pi, g, \alpha$ be as above. A s.a. projection $E \in \pi(G)^{\prime \prime}$ is in $\mathscr{B}(W)^{0^{\prime}}$ iff the measures $\left.\mu_{\pi}\right|_{\tau(\pi, E)} \lambda_{\pi}\left(\left.\mu_{\pi}\right|_{G^{\wedge}-\tau(\pi, E)}\right)$ are singular for all $\lambda \in G^{\wedge}$.

Proof. Since $\pi(G)^{\prime \prime} \subseteq \pi(G)^{\prime}$ and $E, 1-E$ are central projections, the statement follows from Proposition 5.

Now let $G$ be a locally compact group, $\nu$ a finite Borel measure on $G, A \subseteq G$ a Borel set. The measures $g\left(\left.\nu\right|_{A}\right),\left.\nu\right|_{G-A}$ are singular iff there is a Borel set $B \subseteq G$ such that

$$
\left.\nu\right|_{G-A}(B)=\nu(B-A)=0 \quad \text { and } \quad g\left(\left.\nu\right|_{A}\right)(G-B)=\nu\left(A-g^{-1} B\right)=0 .
$$

Definition 4. Let $G, \nu$ be as above. A set $A \subseteq G$ is $\nu$-almost invariant iff for all $g,{ }_{g}\left(\left.\nu\right|_{A}\right),\left.\nu\right|_{G-A}$ are singular. $\nu$ is ergodic iff the only $\nu$-almost invariant sets have $\nu$-measure zero or have complements with $\nu$-measure zero.

Clearly if $\nu$ is discrete then it is ergodic. Also if $\nu$ is equivalent to Haar measure on $G, \nu$ is ergodic. For if $\nu$ is equivalent to Haar measure then any $\nu$ almost invariant set $A$ is equal to an invariant set $B$ (modulo a set of measure zero), and this implies $\nu(B)=0$ or $\nu(G-B)=0$, and so $\nu(A)=0$ or $\nu(G-A)=0$. It is easily verified that the $\nu$-almost invariant Borel sets form a $\sigma$-ring containing the $\nu$-null sets.

Proposition 7. Let $\pi, g, \alpha$ be as above. Then $\mathscr{B}(W)^{0^{\prime}}$ is the $W^{*}$-algebra generated by $\left\{E \in \pi(G)^{\prime \prime}: \tau(\pi, E)\right.$ is $\mu_{\pi}$-almost invariant $\}$.

Proof. Observe $\mathfrak{B}(W)^{0} \supseteq \pi(G)^{\prime}$, so $\mathscr{B}(W)^{0^{\prime}} \subseteq \pi(G)^{\prime \prime}$. The result is then immediate from Proposition 6. 
It is a simple matter to characterize those $\pi$ with no almost periodic operators other than those in $\pi(G)^{\prime}$ : This will be the case iff $\mathscr{B}(W)^{0^{\prime}}=\pi(G)^{\prime \prime}$ which by Proposition 7 is the case iff every Borel set is $\mu_{\pi}$-almost invariant. For $\mu_{\pi}$ discrete this can only occur if $\mu_{\pi}$ is concentrated at one point. It is a consequence of a remarkable theorem of Hewitt and Kakutani that there exists a nonatomic measure $\nu$ on any nondiscrete group such that every Borel set is $\nu$-almost invariant. Suppose $K$ is a nondiscrete L.C.A. group. By the Hewitt-Kakutani theorem [5, 5.2.6] there is an independent Cantor set $L$ in $K$. The facts we shall use about $L$ are that (1) $L$ is a Cantor set and thus carries a nonzero nonatomic measure $\nu$; (2) if $k \in K, k \neq 1, k L \cap L$ contains at most two points. By (2) if $k \neq 1$, then $\left({ }_{k} \nu\right)(L)=\nu\left(k^{-1} L\right)=\nu\left(k^{-1} L \cap L\right)$ $=0$ since $\nu$ is nonatomic. Thus $\nu,{ }_{k} \nu$ are singular and, hence, $\left.\nu\right|_{K-A},{ }_{k}\left(\left.\nu\right|_{A}\right)$ are singular for every Borel set $A \subseteq K$ and every $k \in K$.

From this it follows that any noncompact group has a representation $\pi$ with purely continuous spectral measure and such that $\mathscr{B}(W)^{0}=\pi(G)^{\prime}$.

At the other extreme, $\mu_{\pi}$ is ergodic iff $\mathscr{B}(W)^{0}$ is irreducible. An example of this is the regular representation of $G$. For by Plancherel's theorem the spectral measure of this representation is equivalent to Haar measure on $G^{\wedge}$ and thus is ergodic.

\section{REFERENCES}

1. W. Arveson, On groups of automorphisms of operator algebras, J. Functional Analysis 15 (1974), 217 -243. MR 50 \# 1016.

2. J. Dixmier, Les $C^{*}$-algèbres et leurs représentations, Gauthier-Villars, Paris, 1964. MR 30 \#1404.

3. K. H. Hoffmann, Introduction to the theory of compact groups. I, Lecture Notes, Tulane Univ., $1966-1967$.

4. J. Liukkonen and M. Mislove, Symmetry in Fourier-Stieltjes algebras, Math. Ann. 217 (1975), $97-112$.

5. W. Rudin, Fourier analysis on groups, Interscience, New York, 1962. MR 27 \#2808.

6. H. Schaeffer, Topological vector spaces, Springer-Verlag, New York, 1970.

Instituto de Matemática Pura e Aplicada, Rua luiz de Camões 68, Rio de Janeiro, BRAZIL 